\title{
Intra-arterial chemotherapy in locally advanced or recurrent carcinomas of the penis and anal canal: an active treatment modality with curative potential
}

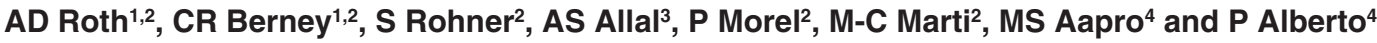 \\ ${ }^{1}$ Oncosurgery; ${ }^{2}$ Department of Surgery; ${ }^{3}$ Division of Radio-oncology; ${ }^{4}$ Division of Oncology, Geneva University Hospital, 24 Micheli-du-Crest, \\ 1211 Geneva 14, Switzerland
}

Summary The prognosis of locally advanced or recurrent carcinomas of the penis (PE) and of the anal canal (AC) after conventional treatment is dismal. We report 16 patients (eight with AC carcinomas and eight with PE cancers) treated by intra-arterial (IA) chemotherapy. Fifteen of them were treated for locally advanced or recurrent disease and one in an adjuvant setting. The chemotherapy was administered via a femoral IA catheter with its tip located above the aortic bifurcation, under the inferior mesenteric artery. It consisted of eight push injections, given over a 48-h period, of the following drug combination: cisplatin $8.5 \mathrm{mg} \mathrm{m}^{-2}, 5-\mathrm{FU} 275 \mathrm{mg} \mathrm{m}^{-2}$, methotrexate $27.5 \mathrm{mg} \mathrm{m}^{-2}$, mitomycin C $1.2 \mathrm{mg} \mathrm{m}^{-2}$, and bleomycin $4 \mathrm{mg} \mathrm{m}^{-2}$. Leucovorin was given po, $4 \times 15 \mathrm{mg} \mathrm{day}^{-1}$, during the chemotherapy and for 3 days thereafter. A total of 52 cycles of treatment were administered. Of the 15 patients evaluable for response, six obtained a CR (three PE, three $\mathrm{AC}$ ) and eight a PR. Among the complete responders, four are alive and disease-free 2-15 years after treatment. The other patients enjoyed an objective response lasting 3-25 months (median 7 months). Four patients developed grade III/IV haematological toxicity with three episodes of febrile neutropenia, one of them with a fatal outcome due to patient's failure to obtain medical attention at the onset of his fever, one a grade III mucositis of the glans, and four a grade III/IV cutaneous toxicity, the latter caused by the IA administration of bleomycin. In conclusion, IA chemotherapy is effective and potentially curative in locoregionally advanced or recurrent carcinomas of the penis and of the anus. Its contribution in the primary management of advanced penile or anal carcinoma should be prospectively investigated. (C) 2000 Cancer Research Campaign http://www.bjcancer.com

Keywords: penile cancer; anal canal cancer; intra-arterial chemotherapy; intra-arterial bleomycin

Uncontrolled carcinomas of the anal canal (AC) and of the penis (PE) share in common the capacity to infiltrate the perineum and the lower pelvis, leading to locoregional complications and intractable morbidity. Despite the important progress accomplished in the treatment of AC carcinoma, with a cure rate up to $70-75 \%$ even in patients with locally advanced lesions, about $25 \%$ of the patients with T3-4 tumours end up with intractable locoregional disease (Gerard et al, 1995; Flam et al, 1996; UKCCCR Anal Cancer Trial Working Party, 1996; Ryan et al, 2000). In PE cancer, apart from the cases presenting with early lesions (T1-2, N0, M0), the prognosis is poor and more than $60 \%$ of patients with node-positive disease suffer from uncontrolled perineal invasion, despite additional surgical resections and radiation therapy (Connell and Berger, 1994). Furthermore, the successive use of surgery and radiotherapy over the inguinal areas can lead to untractable leg oedema in a significant number of cases.

Once the disease escapes local therapeutic modalities little can be done. This fact is witnessed by the lack of pertinent literature on the subject. Systemic chemotherapy has been shown to have some activity in both diseases. Unfortunately, it is mostly transient, especially in relapsing, heavily-pretreated perineal disease (Wilking et al, 1985; Ajani et al, 1989; Eisenberger, 1992; Connell and Berger, 1994).

Received 27 March 2000

Revised 26 July 2000

Accepted 9 August 2000

Correspondence to: AD Roth
In order to deliver higher concentrations of drugs to the affected area, intra-arterial (IA) administration of chemotherapy has been attempted in several neoplastic situations such as isolated liver metastasis from colorectal carcinoma and neuroendocrine tumours, as well as relapsing gynaecologic cancers (Stephens et al, 1980; Collins, 1984; Kavanagh, 1984; Scarabelli et al, 1987; Patt and Mavligit, 1991). Most results reported show that IA chemotherapy induces a higher response rate than systemic chemotherapy, although its impact on overall survival is still controversial (Patt, 1993).

Uncontrolled perineal infiltration by both $\mathrm{AC}$ and PE cancers is a situation in which a higher impact on the disease to alleviate symptoms is the main issue, while overall survival benefits are of minor interest. IA chemotherapy, with its usual higher response rate than systemic therapy, could therefore be a useful option in these circumstances where nothing else can be offered. An IA regimen combining most of the drugs known to be active in these diseases was designed and experience in sixteen patients with advanced PE and AC tumours is presented.

\section{PATIENTS AND METHODS}

Sixteen patients with advanced localized AC (eight cases) and PE (eight cases) cancers treated from April 1983 to December 1998 are retrospectively analysed. There were 10 men and six women, age range 35-83 years. All patients had histologically confirmed recurrent (six PE, five AC) or locally advanced (two PE, three AC) malignancies. All but two patients with PE carcinoma refused 
primary penile amputation. In these two latter cases, IA treatment was given as an additional therapy for residual pelvic disease found after partial penectomy with bilateral superficial inguinal lymphadenectomy.

IA pelvic infusion was performed using a Pigtail type $5 \mathrm{~F}$ catheter introduced percutaneously into the femoral artery and passed retrograde until the tip was just above the bifurcation of the aorta. Pelvic arteriograms were performed using $30 \mathrm{ml}$ Optiray administration to check catheter placement. The catheter was kept inserted for the $48 \mathrm{~h}$ duration of treatment. Its position was rechecked once at $24 \mathrm{~h}$ by angioscintigraphy. In one patient with AC carcinoma (patient no.10), the catheter had to be introduced into the humeral artery, due to major oedema of the lower limbs reaching up to the pelvis and making catheterization of the femoral artery impossible.

The treatment consisted of the IA administration of cisplatin (CDDP) $8.5 \mathrm{mg} \mathrm{m}^{-2}$, 5-FU $275 \mathrm{mg} \mathrm{m}^{-2}$, methotrexate (MTX) $27.5 \mathrm{mg} \mathrm{m}^{-2}$, Mitomycin C $1.2 \mathrm{mg} \mathrm{m}^{-2}$, and bleomycin (BLM) $4 \mathrm{mg} \mathrm{m}^{-2}$, given eight times in rapid push injections over a period of $48 \mathrm{~h}$. In patient no.10, who was treated through the humeral artery, the eight injections were administered over $24 \mathrm{~h}$, in order to limit the risk of thrombosis. Leucovorin was given orally during the treatment and the three following days at $4 \times 15 \mathrm{mg} \mathrm{day}^{-1}$. The treatment courses were given at 4-5-week intervals; the total number of courses varied depending on patient tolerance. Since April 1995, BLM was not given IA any more because of intolerable cutaneous toxicity. It was replaced by the iv administration of $10 \mathrm{mg}$ of BLM on days 1,8 and 15 of each treatment cycle. Patients older than 75 years did not receive MTX and received only six IA injections per cycle (25\% dose-reduction of the total dose of all drugs).

\section{RESULTS}

The clinical status, treatment and outcome of eight patients with $\mathrm{PE}$ are summarized in Table 1. Among the seven patients evaluable for response, three achieved a complete response (CR) and three a partial response (PR). The remaining patient evaluable for response (patient no. 7) experienced clinical improvement (disappearance of left-leg oedema and left inguinal tumoural infiltration) without meeting the radiological criteria for a PR. He is therefore reported as stable disease (SD). Among the patients with CR, two remain disease-free more than 5 and 12 years later. One of them (patient no. 2) had limited surgery consisting in a circumcision followed 6 months later by IA therapy for a biopsy-proven relapse in the left inguinal lymph nodes. A new surgical sampling of the left groin after treatment completion failed to show any sign of tumour and the patient is still disease-free 15 years later. The second patient (patient no. 8) underwent a more complete surgery as primary treatment in July 1993, consisting in a partial penectomy and partial lymphadenectomy on both sides. One lymph node on the right was positive and the disease was therefore staged pT1pN1G3. In October 1993 an excision biopsy performed in the left groin was positive for tumour. In December 1993 he developed a $3 \mathrm{~cm}$ diameter tumoural node on the left, as well as a suspicious enlarged node on the right, and was started on IA chemotherapy. After four cycles of treatment the patient underwent bilateral inguinal surgery and was found in pathological CR. He received two additional courses of IA chemotherapy and is still in complete remission 5 years later.
Table 2 reports the clinical status treatment and outcome of eight patients with AC. Three patients (no. 12, 14 and 16) received IA chemotherapy in a neoadjuvant setting before classical chemoradiation (5-FU plus MitoC, 55 Gy) because of very advanced locoregional disease. One of them (no. 16) received only limited chemoradiation (36.8 Gy) due to previous irradiation for cervix cancer 10 years before. All three patients responded completely to the therapeutic programme and two are still in CR after 32 months and 4 years of follow-up, respectively. The five other patients were treated for uncontrolled perineal relapse after failure of chemoradiation. Three of them failed secondary surgery consisting of an abdomino-perineal amputation. All five patients responded to therapy and enjoyed a remission of at least 6 months. One of them completed a long-standing CR lasting for more than 8 years.

A total of 52 courses of IA chemotherapy were administered and are evaluable for toxicity. Grade III and IV toxicities are summarized in Table 3. Four patients had one occurrence of grade III-IV haematological toxicity (leukopenia) and three of them had to be hospitalized once for an episode of febrile neutropenia (patients no. 9, no. 11 and no. 14), one of which was fatal due to failure to obtain medical attention the day of fever onset (patient no. 14). One patient presented grade III mucositis of the glans. One patient (patient no. 1) developed peripheral thromboembolism to the right lower limb which did not necessitate any surgical procedure. In one case (patient no. 13), treatment had to be discontinued due to grade III asthenia following the first cycle of IA chemotherapy. She was one of the oldest patients treated in this series.

A cutaneous toxicity was observed in nine patients. Among them five suffered from grade III-IV cutaneous lesions, limiting the treatment to a maximum of three cycles per patient. It consisted in the development of a painful diffuse livedo reticularis and of macular erythematous lesions, centred by violaceous areas, evolving in some cases to cutaneous ulcerations and necrosis (Figure 1). The cutaneous toxicity was generally limited to the thighs and scrotum, was cumulative, and, in the absence of apparent necrosis, resolved in 2-3 weeks. It was seen only in conjunction with IA administration of bleomycin (BLM), since none of the patients not exposed to IA BLM ever developed such cutaneous lesions. Furthermore, two patients (no. 6 and no. 9) who initially developed significant cutaneous toxicity with IA BLM did not develop any new cutaneous lesions when the treatment was resumed without this drug.

\section{DISCUSSION}

The results show that IA chemotherapy is very efficient in PE and AC tumours. All heavily pretreated patients responded to it and some of them remained permanently disease-free (patients no. 8 and 9). One patient with PE cancer who refused classical treatment was even cured per primam by local excision and IA chemotherapy only (patient no. 2). It is interesting to note that IA chemotherapy was still active in all patients even in areas pretreated with radiation therapy. This might be the result of better drug penetration in vascularly compromised tumoural areas. Response was usually very quick to occur, sometimes after only one cycle of treatment, as shown in Figure 2 (patient no. 15).

Apart from febrile neutropenias which occurred in four instances, with one fatality due to patient's failure to obtain medical attention, the cutaneous toxicity was the most striking toxicity and 


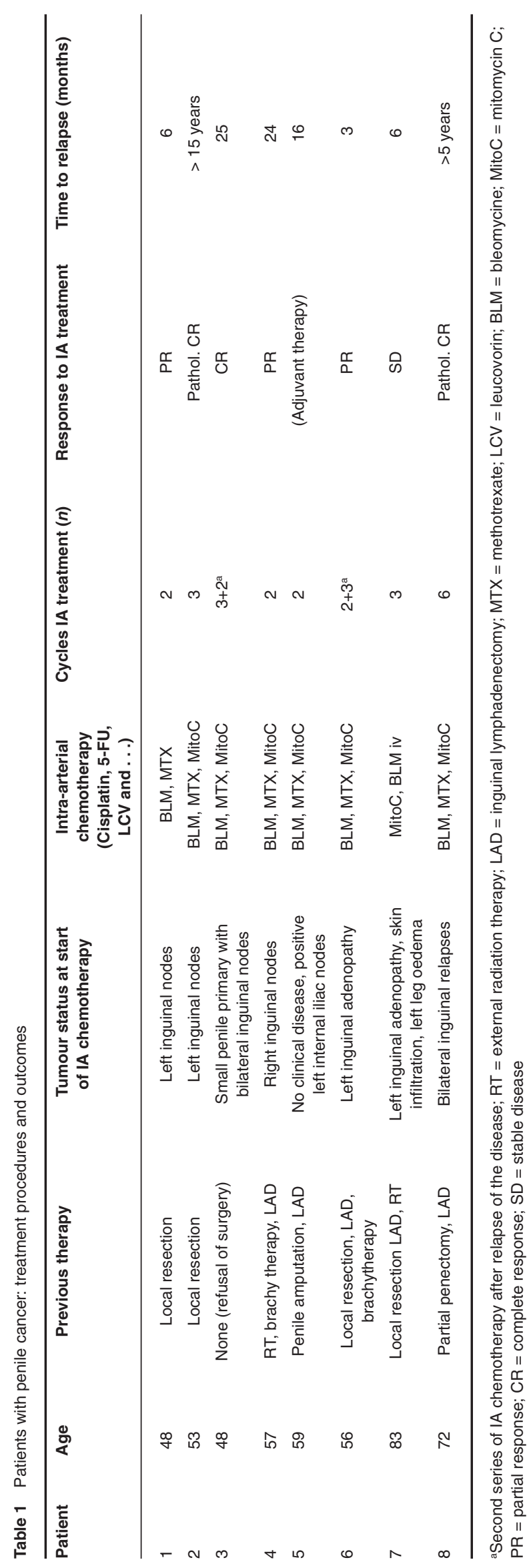




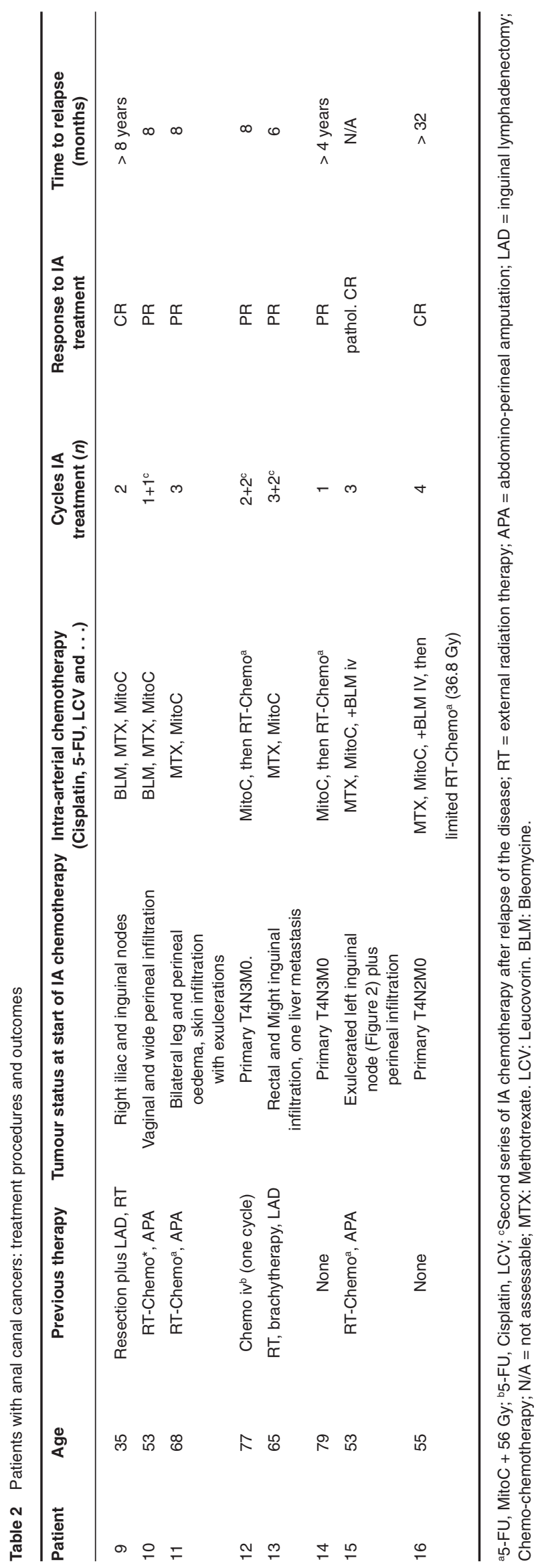

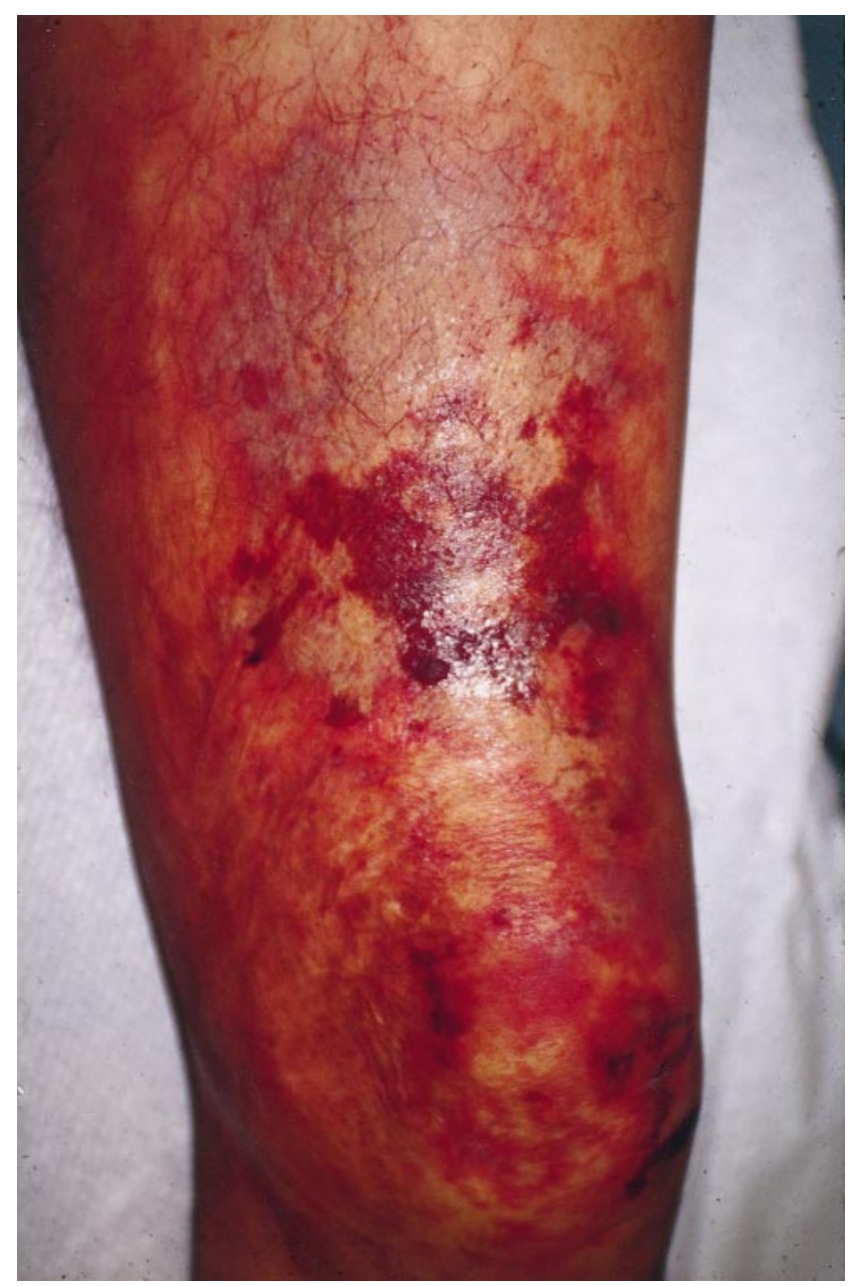

Figure 1 Grade IV cutaneous toxicity after two cycles of IA chemotherapy containing bleomycin (patient no. 6)

responsible for interruption of the treatment in many instances. It was finally attributed to BLM given IA, and no significant cutaneous toxicity occurred after cessation of the IA administration of this drug.

BLM is much better known for its pulmonary toxicity which can be life-threatening because of its evolution into lung fibrosis (Swenerton et al, 1979; Morrow et al, 1977). BLM administered iv can elicit an erythema of the skin leading to cutaneous pigmentation without major consequences. Severe cutaneous toxicity that can lead to skin necrosis, however, has been reported only once by others following IA administration of BLM (Morrow et al, 1977). It is well demonstrated that BLM has a direct action on endothelial

Table 3 Grade III-IV toxicity and complications

\begin{tabular}{ll}
\hline Related to chemotherapy (52 cycles) & \\
Leukopenia & 4 \\
Cutaneous & 5 \\
Mucitis & 1 \\
Fatal sepsis & 1 \\
Related to catheterization & 1 \\
$\quad$ Arterial emboli & \\
Other & 1 \\
$\quad$ Severe asthenia & \\
\hline
\end{tabular}



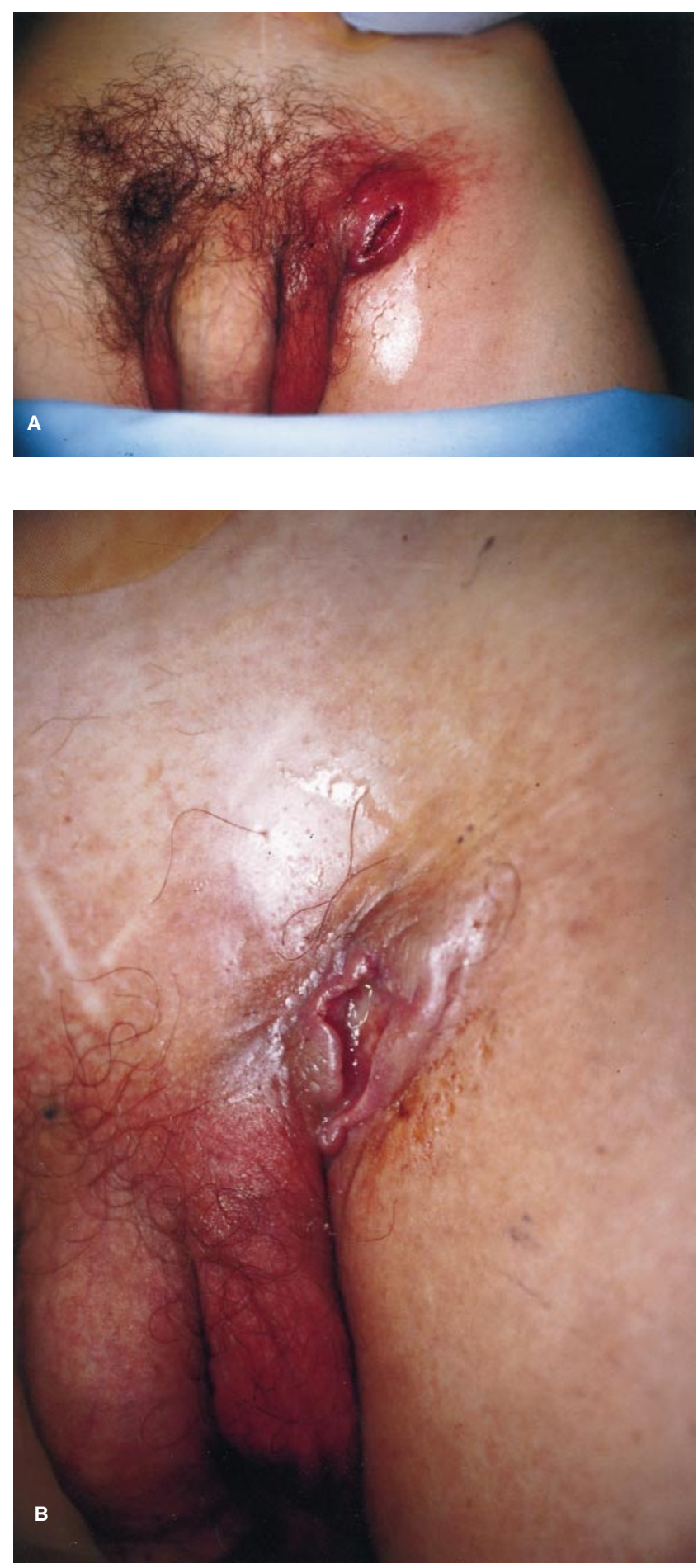

cells by production of oxidant agents (Adamson and Bowden, 1974; Lazo and Humphreys, 1983). Raynaud's phenomenon has sometimes been observed following BLM iv administration and is believed to be a clinical manifestation of BLM toxicity to the endothelium (Vogelzang et al, 1981; Cohen et al, 1973; Smith et al, 1985). The location of the skin lesions close to the IA delivery site of the drug suggests that the concentration of BLM at this level may be toxic to the skin capillaries.

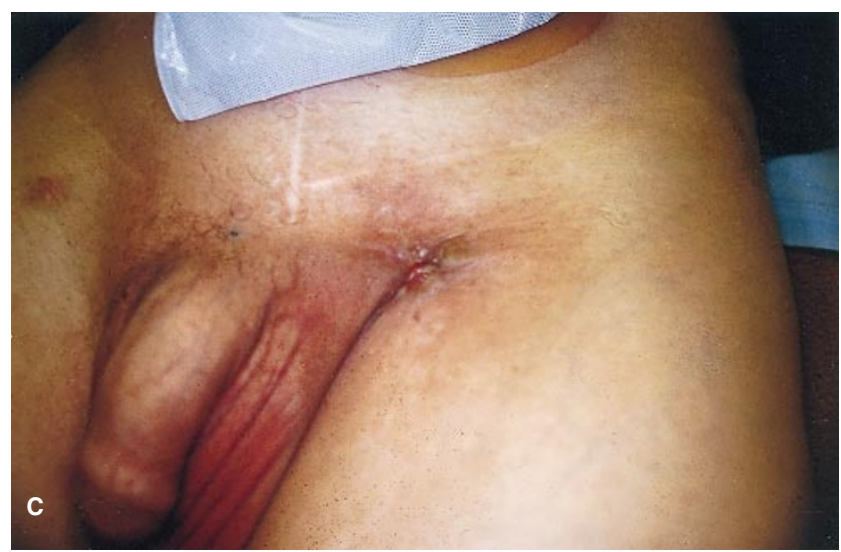

Figure 2 Left inguinal relapse of a heavily pretreated carcinoma of the anal canal (patient no. 15) Status before start of IA treatment, $(\mathbf{A})$ after 1 cycle of treatment, (B) and after two cycles $(\mathbf{C})$

Since BLM is a major drug, especially in PE (Edsmyr et al, 1985; Eisenberger, 1992), we are now giving it by iv route, concomitantly with IA chemotherapy. So far no cutaneous toxicity $>$ grade 1 has been elicited, indicating that this side-effect is not the result of the interaction of BLM with one of the other chemotherapeutic agents still given IA.

Because a large proportion of AC patients are of geriatric age, it is of interest to note that three of the treated patients were in their late seventies and early eighties. All received the chemotherapy with $25 \%$ dose-reduction (six IA injections instead of eight) and the methotrexate was omitted. All developed manageable toxicity without late side-effects. Thus, we think that in selected cases IA chemotherapy can be reasonably proposed in elderly patients.

In conclusion, these results show that IA chemotherapy is an useful therapeutic modality in advanced stage and relapsing PE and $\mathrm{AC}$, and that it is curative in some selected cases. It has manageable toxicity and can even be safely administered in geriatric patients. IA chemotherapy should be considered in all patients presenting with locoregionally relapsing PE and AC. Its integration in the primary treatment of patients presenting with advanced lesions, for which it can be forseen that conventional treatment would yield little chance of cure, should be considered and tested in larger series.

\section{REFERENCES}

Adamson IY and Bowden DH (1974) The pathogenesis of bleomycin-induced pulmonary fibrosis in mice. Am J Pathol 77: 185-198

Ajani JA, Carrasco CH, Jackson DE and Wallace S (1989) Combination of cisplatin plus fluoropyrimidine chemotherapy effective against liver metastases from carcinoma of the anal canal. Am J Med 87: 221-224

Cohen IS, Moseher MB, O'keefe EJ, Klaus SN and DeConti RC (1973) Cutaneous toxicity of bleomycin therapy. Arch Dermatol 107: 553-555

Collins JM (1984) Pharmacologic rationale for regional drug delivery. J Clin Oncol 2: $498-504$

Connell CF and Berger NA (1994) Management of advanced squamous cell carcinoma of the penis. Urol Clin North Am 21: 745-756

Edsmyr F, Andersson L and Esposti PL (1985) Combined bleomycin and radiation therapy in carcinoma of the penis. Cancer 56: 1257-1263

Éisenberger MA (1992) Chemotherapy for carcinomas of the penis and urethra. Urol Clin North Am 19: 333-338

Flam M, John M, Pajak TF, Petrelli N, Myerson R, Doggett S, Quivey J, Rotman M, Kerman H, Coia L, and Murray K (1996) Role of mitomycin in combination 
with fluorouracil and radiotherapy, and of salvage chemoradiation in the definitive nonsurgical treatment of epidermoid carcinoma of the anal canal: results of a phase III randomized intergroup study. J Clin Oncol 14: 2527-2539

Gerard JP, Romestaing P, Ardiet JM, Trillet Lenoir V, Rocher FP, Baron MH, and Buatois F (1995) Current treatment of cancers of the anal canal. Ann Chir 49: 363-368

Kavanagh JJ, Jr. (1984) Regional chemotherapeutic approaches to the management of pelvic malignancies. Cancer Bull 36: 52-55

Lazo JS and Humphreys CJ (1983) Lack of metabolism as the biochemical basis of bleomycin-induced pulmonary toxicity. Proc Natl Acad Sci USA 80: 3064-3068

Morrow CP, Di Saia PJ, Mangan CF, and Lagasse LD (1977) Continuous pelvic arterial infusion with bleomycin for squamous carcinoma of the cervix recurrent after irradiation therapy. Cancer Treat Rep 61: 1403-1405

Patt YZ and Mavligit GM (1991) Arterial chemotherapy in the management of colorectal cancer: an overview. Semin Oncol 18: 478-490

Patt YZ. (1993) Regional hepatic arterial chemotherapy for colorectal cancer metastatic to the liver: the controversy continues. J Clin Oncol 11: 815-819

Ryan DP, Compton CC, and Mayer RJ (2000) Carcinoma of the anal canal. $N$ Engl J Med 342: 792-800

Scarabelli C, Tumolo S, De Paoli A, Frustaci S, Campagnutta E, Morassut S, Franchin G, Crivellari D, Sopracordevole F, Lo Re G and et al (1987) Intermittent pelvic arterial infusion with peptichemio, doxorubicin, and cisplatin for locally advanced and recurrent carcinoma of the uterine cervix. Cancer 60: 25-30

Smith EA, Harper FE, and Le Roy EC (1985) Raynaud's phenomenon of a single digit following local intradermal bleomycin sulfate injection. Arthritis Rheum 28: $459-461$

Stephens FO, Harker GJ, and Crea P (1980) The intraarterial infusion of chemotherapeutic agents as 'basal' treatment of cancer: evidence of increased drug activity in regionally infused tissues. Aust NZ J Surg $\mathbf{5 0}$ : $597-602$

Swenerton KD, Evers JA, White GW, and Boyes DA (1979) Intermittent pelvic infusion with vincristine, bleomycin, and mitomycin $\mathrm{C}$ for advanced recurrent carcinoma of the cervix. Cancer Treat Rep 63: 1379-1381

UKCCCR Anal Cancer Trial Working Party. UK Coordinating Committee on Cancer Research. (1996) Epidermoid anal cancer: results from the UKCCCR randomised trial of radiotherapy alone versus radiotherapy, 5-fluorouracil, and mitomycin. Lancet 348: 1049-1054

Vogelzang N, Bosl G, Johnson K, and Kennedy BJ (1981) Raynaud's phenomenon: a common toxicity after combination chemotherapy for testicular cancer. Ann Intern Med 95: 288-292

Wilking N, Petrelli N, Herrera L, and Mittelman A. (1985) Phase II study of combination bleomycin, vincristine and high-dose methotrexate (BOM) with leucovorin rescue in advanced squamous cell carcinoma of the anal canal. Cancer Chemother Pharmacol 15: 300-302 